\title{
BMJ Open Pharmacy Diabetes Screening Trial: protocol for a pragmatic cluster- randomised controlled trial to compare three screening methods for undiagnosed type 2 diabetes in Australian community pharmacy
}

\author{
Ines Krass, ${ }^{1}$ Rob Carter, ${ }^{2}$ Bernadette Mitchell, ${ }^{1}$ Mohammadreza Mohebbi, ${ }^{3}$ \\ Sophy T F Shih, ${ }^{2}$ Peta Trinder, ${ }^{4}$ Vincent L Versace, ${ }^{5}$ Frances Wilson, ${ }^{1}$ \\ Kevin Mc Namara ${ }^{4}$
}

To cite: Krass I, Carter R, Mitchell B, et al. Pharmacy Diabetes Screening Trial: protocol for a pragmatic cluster-randomised controlled trial to compare three screening methods for undiagnosed type 2 diabetes in Australian community pharmacy. BMJ Open 2017;7:e017725. doi:10.1136/ bmjopen-2017-017725

- Prepublication history for this paper is available online. To view these files, please visit the journal online (http://dx.doi. org/10.1136/bmjopen-2017017725).

Received 16 May 2017 Revised 19 September 2017 Accepted 28 September 2017

CrossMark

For numbered affiliations see end of article.

Correspondence to

Professor Ines Krass; ines.krass@sydney.edu.au

\begin{abstract}
Introduction With the rising prevalence of type 2 diabetes in Australia, screening and earlier diagnosis is needed to provide opportunities to intervene with evidence-based lifestyle and treatment options to reduce the individual, social and economic impact of the disease. The objectives of the Pharmacy Diabetes Screening Trial are to compare the clinical effectiveness and cost-effectiveness of three screening models for type 2 diabetes in a previously undiagnosed population.

Methods and analysis The Pharmacy Diabetes Screening Trial is a pragmatic cluster randomised controlled trial to be conducted in 363 community pharmacies across metropolitan, regional and remote areas of Australia, randomly allocated by geographical clusters to one of three groups, each with 121 pharmacies and 10304 screening participants. The three groups are: group A: risk assessment using a validated tool (AUSDRISK); group B: AUSDRISK assessment followed by point-of-care glycated haemoglobin testing; and group C: AUSDRISK assessment followed by point-ofcare blood glucose testing. The primary clinical outcome

Strengths and limitations of this study

- The study will include a nationally representative sample of both pharmacies and the population at risk of type 2 diabetes.

- The results will inform the potential real-world effectiveness of each intervention at a population level.

- Notwithstanding standardised approaches and clear documentation for data collection, some data obtained from pharmacists may be inaccurate or incomplete.

- Recruitment of screening participants by pharmacists may not achieve the expected uptake.

- Verification of whether there has been a diagnosis of type 2 diabetes mellitus or pre-diabetes may be impossible for a small proportion of referred screening participants whose diabetes testing does not trigger National Diabetes Support Scheme registration and where follow-up with the patient, pathology lab and general practitioner cannot be achieved or otherwise fails to confirm an outcome.
\end{abstract} measure is the proportion of newly diagnosed cases of type 2 diabetes. Primary outcome comparisons will be conducted using the Cochran-Mantel-Haenszel test to account for clustering. The secondary clinical outcomes measures are the proportion of those who (1) are referred to the general practitioner (GP), (2) take up referral to the GP, (3) are diagnosed with pre-diabetes, that is, impaired glucose tolerance or impaired fasting glucose and (4) are newly diagnosed with either diabetes or prediabetes. The economic outcome measure is the average cost (direct and indirect) per confirmed new case of diagnosed type 2 diabetes based on the incremental net trial-based costs of service delivery and the associated incremental longer term health benefits from a health funder perspective.

Ethics and dissemination The protocol has been approved by the Human Research Ethics Committees at University of Sydney and Deakin University. Results will be available on the Sixth Community Pharmacy Agreement website and will be published in peer reviewed journals. Trial registration number ACTRN12616001240437; Preresults.

\section{BACKGROUND}

Type 2 diabetes mellitus (T2DM) is a chronic metabolic disorder characterised by the progressive failure of insulin secretion and/or increasing resistance of body tissues to insulin resulting in high blood glucose levels (hyperglycaemia). ${ }^{1}$ In Australia, the estimated prevalence of diagnosed T2DM increased from $1.5 \%$ in $1989 / 1990$ to $4.4 \%$ in $2014 / 2015 .^{23}$ 
Currently, there are approximately 1.2 million Australians with diagnosed T2DM and an estimated further 500000 with undiagnosed T2DM. ${ }^{4}$ In addition, almost one in six Australian adults (more than 2 million inintdividuals) over the age of 25 years is affected by pre-diabetes, that is, impaired glucose tolerance (IGT) or impaired fasting glucose (IFG), in which blood glucose levels are elevated but not high enough to be diagnosed with T2DM. ${ }^{6}$ Undetected diabetes or pre-diabetes have implications for the health system and for individuals who are unaware of their condition and consequently do not have the opportunity to reduce their risk of developing chronic conditions caused by uncontrolled hyperglycaemia, including macrovascular complications (eg, peripheral vascular disease, myocardial infarction, cardiovascular disease and stroke) and microvascular complications (eg, diabetic kidney disease, peripheral sensory neuropathy and diabetic retinopathy). ${ }^{6-10}$ There is often a latent period of many years with no symptoms before the disease is diagnosed, and comorbidities and complications may already have developed at diabetes diagnosis. ${ }^{11} 12$

Studies have shown that the progression of diabetes and its complications can be reduced or delayed by lifestyle changes and pharmacotherapy. ${ }^{13-15}$ These findings highlight the need for more effective screening of the general population to facilitate increased risk identification and effective early management to reduce the risk of developing T2DM and its complications.

Many risk factors for T2DM are increasing in prevalence in line with increasing age and obesity. Since 1994, the proportion of Australia's population aged 65 years and over increased from $11.8 \%$ to $14.7 \% .^{3}$ In 2014-2015, 11.2 million (63.4\%) of Australians over the age of 18 years were overweight or obese and this increased with geographic remoteness and lower socioeconomic status. ${ }^{3}$ Certain ethnicities have a higher prevalence of T2DM; Pacific Islander, southern European or Asian backgrounds are twice as likely, and Aboriginal and Torres Strait Islanders four times more likely, compared with other Australians, to have developed diabetes within 5 years based on self-report and measured data. ${ }^{16-18}$

The social, human and economic impact of T2DM in Australia has led to the development of the Australian National Diabetes Strategy. ${ }^{19}$ This strategy aims to coordinate existing healthcare resources across all levels of government to reduce the impact of diabetes in the community. One such resource is community pharmacy. A recent review of pharmacy-based screening programmes reported that community pharmacies are feasible sites for screening and that a significant number of risk factors, such as high blood pressure, cholesterol and diabetes risk, were correctly identified in community pharmacies. ${ }^{20}$ There are more than 5000 community pharmacies in Australia with an extensive distribution throughout metropolitan, regional and remote areas. ${ }^{21}$ In addition, the overwhelming majority (94\%) of Australians reported using a pharmacy in 2012, ${ }^{22}$ providing an opportunity to engage people along the health spectrum, including hard-to-reach populations who do not use other health services.

Australian pharmacies currently use a variety of means to test for diabetes and diabetes risk, including the use of AUSDRISK, a 10-item questionnaire that has been validated to predict risk of progression to T2DM over a 5-year period in a diverse ethnic population. It includes questions based on age, sex, ethnicity, family history of diabetes, history of abnormal glucose metabolism, smoking status, current hypertensive treatment, physical activity, fruit and vegetable consumption and waist circumference to generate a risk score.

Other methods include random and fasting small capillary blood glucose testing (scBGT) and, to a lesser extent, with point-of-care (POC) glycated haemoglobin (HbAlc) testing. The addition of a subsequent blood glucose test for those with AUSDRISK score $\geq 12$ has been shown to be a more effective approach to screening, compared with using the AUSDRISK alone. ${ }^{23-26}$

Despite mounting evidence to support the feasibility and value of pharmacy as a component of population screening efforts, few studies, to date, have sought to determine the comparative effectiveness or cost-effectiveness of different screening intervention options. ${ }^{23} 24$ As such, the optimal approach to pharmacy screening remains uncertain. The Pharmacy Diabetes Screening Trial (PDST) aims to compare the diagnostic and economic outcomes for several evidence-based and pragmatic models of diabetes screening in a community pharmacy setting.

An expert panel consisting a range of stakeholders with expertise in diabetes, including representatives from The Boden Institute at University of Sydney; Australian Diabetes Society; Australian Diabetes Educators Association; Royal Australian College of General Practitioners (RACGP); Pharmaceutical Society of Australia; Community Pharmacy; PDST Research team; Diabetes Australia; Department of Health; and Trials Advisory Group has been established to provide guidance on (1) the trial design; (2) communication strategies; and (3) how to support the engagement, recruitment and retention of participants (4) trial monitoring.

\section{Trial objectives}

The objectives of the PDST are to compare the effectiveness and cost-effectiveness of three different pharmacy-based screening models:

1. AUSDRISK alone (group A)

2. AUSDRISK followed by a POC test for HbA1c (group B)

3. AUSDRISK followed by a POC scBGT (group C).

\section{Hypotheses}

The primary null hypothesis is that there will be no differences between groups A and B or C in the proportion of participants who are newly diagnosed with diabetes. 
The secondary null hypotheses are that there will be no differences between groups $\mathrm{A}$ and $\mathrm{B}$ or $\mathrm{C}$ in the proportion of patients who are diagnosed with diabetes or pre-diabetes, are referred and take up referral to the general practitioner (GP) as well as the average costs (direct and indirect) of service delivery and incremental longer health benefits from a health funder perspective.

The primary clinical hypothesis to be tested is that the addition of either an HbA1c POC test (group B) or an scBGT POC test (group C) to the AUSDRISK assessment will be associated with a statistically significant increase in the proportions of newly diagnosed T2DM cases compared with AUSDRISK alone (group A).

Additional clinical hypotheses related to the primary hypothesis are that groups B and $\mathrm{C}$ will be associated with a statistically significant decrease in the proportions of those who are referred to the GP, take up referral with the GP, are newly diagnosed with pre-diabetes, that is, IFG or IGT, and are newly diagnosed with either diabetes or pre-diabetes compared with group A.

The economic hypotheses to be tested will take a health service funder perspective and involve both trialbased and modelled components. They are: (1) that the addition of either HbA1c POC test or scBGT POC test to AUSDRISK screening is cost-effective in comparison with AUSDRISK screening alone, having regard to trial-based costs and clinical outcomes; (2) that adding either HbAlc POC test or scBGT POC test 'dominates' AUSDRISK screening alone, having regard to modelled longer term health and patient outcomes; and (3) that the cost impact of adding POC testing to AUSDRISK screening is more than offset by the reduction in GP-based costs (fall in false positives in trial) and lower treatment costs (modelled health outcomes).

\section{METHODS}

\section{Trial design}

The PDST uses a cluster randomised controlled design where pharmacies (clusters) are the unit of randomisation, and screening participants are the unit of analysis. To ensure the representativeness of the pharmacy sample, a geographical method will be used to recruit 363 pharmacies in all states and territories in Australia to take account of population density, residential distribution and socioeconomic profile of communities ${ }^{27}$ (table 1). This method will involve data triangulation from several sources, including:

- a national database of pharmacies with accreditation from the Quality Care Pharmacy Program ${ }^{28}$

- postcode boundaries ${ }^{29}$

- socioeconomic indexes for areas (SEIFA) scores and deciles from the 2011 (or later on availability) census ${ }^{30}$

- remoteness areas. ${ }^{31}$

Residential location stratification will be achieved by reclassification of the ABS remoteness areas from five to
Table 1 Statewide distribution of the pharmacies according to residential location

\begin{tabular}{lcccc}
\hline State or territory & Metro & Regional & Remote & Total \\
\hline New South Wales & 80 & 25 & 3 & 108 \\
Victoria & 59 & 25 & 2 & 86 \\
Queensland & 39 & 28 & 5 & 72 \\
South Australia & 14 & 9 & 7 & 30 \\
Western Australia & 22 & 6 & 6 & 34 \\
\hline Tasmania & 0 & 11 & 3 & 14 \\
Northern Territory & 0 & 7 & 4 & 11 \\
\hline Australian Capital & 8 & 0 & 0 & 8 \\
Territory & & & & \\
Australia total & 222 & 111 & 30 & 363 \\
\hline
\end{tabular}

Metro represents the Australian Bureau of Statistics (ABS) category: 'Major Cities of Australia'. Regional represents the ABS categories: 'Inner Regional Australia' and 'Outer Regional Australia'. Remote represents the ABS categories: 'Remote Australia' and 'Very Remote Australia'. Tasmania and Northern Territory will have their allocation of Metropolitan moved to Regional due to the absence of metropolitan areas in these locations.

three categories as follows: the ABS categories of 'Inner Regional Australia' and 'Outer Regional Australia' will be aggregated to a single 'Regional' category, and the ABS categories of 'Remote Australia' and 'Very Remote Australia' will be aggregated to a single 'Remote' category. Sampling proportions for 'Major Cities' (metropolitan) areas of Tasmania and Northern Territory will be reallocated to Regional due to the absence of metropolitan areas in these jurisdictions.

Geocoded pharmacy locations will be used to extract the postcode, the SEIFA scores and remoteness area using a spatial join. For each stratum (ie, state or territory by remoteness classification shown in table 1), summary statistics that provide a description of the socioeconomic status conditions across that stratum (eg, major cities of New South Wales) will be generated. Each stratum will be sorted by postcode in descending order and three sequences of postcodes selected. Selecting sequences of postcodes typically yields contiguous groups representative of the stratum from which they are selected. All data will be applied at a postcode level.

Pharmacies will be selected from geographical groups of colocated postcodes (clusters) and randomly allocated to groups A, B or C, each with 121 pharmacies (figure 1). The advantage of this is that colocated pharmacies within a cluster represent a local community that will all receive the same service model to reduce the contamination bias between groups.

\section{Sample size-screening participants}

Sample size calculations will be based on a T2DM incidence of $4.6 \%^{32}$ and allowing for incidence to range from $1.4 \%$ to $8.5 \%$ across the age groups. ${ }^{32}$ The CochranMantel-Haenszel (CMH) method ${ }^{33}$ will be used for the 


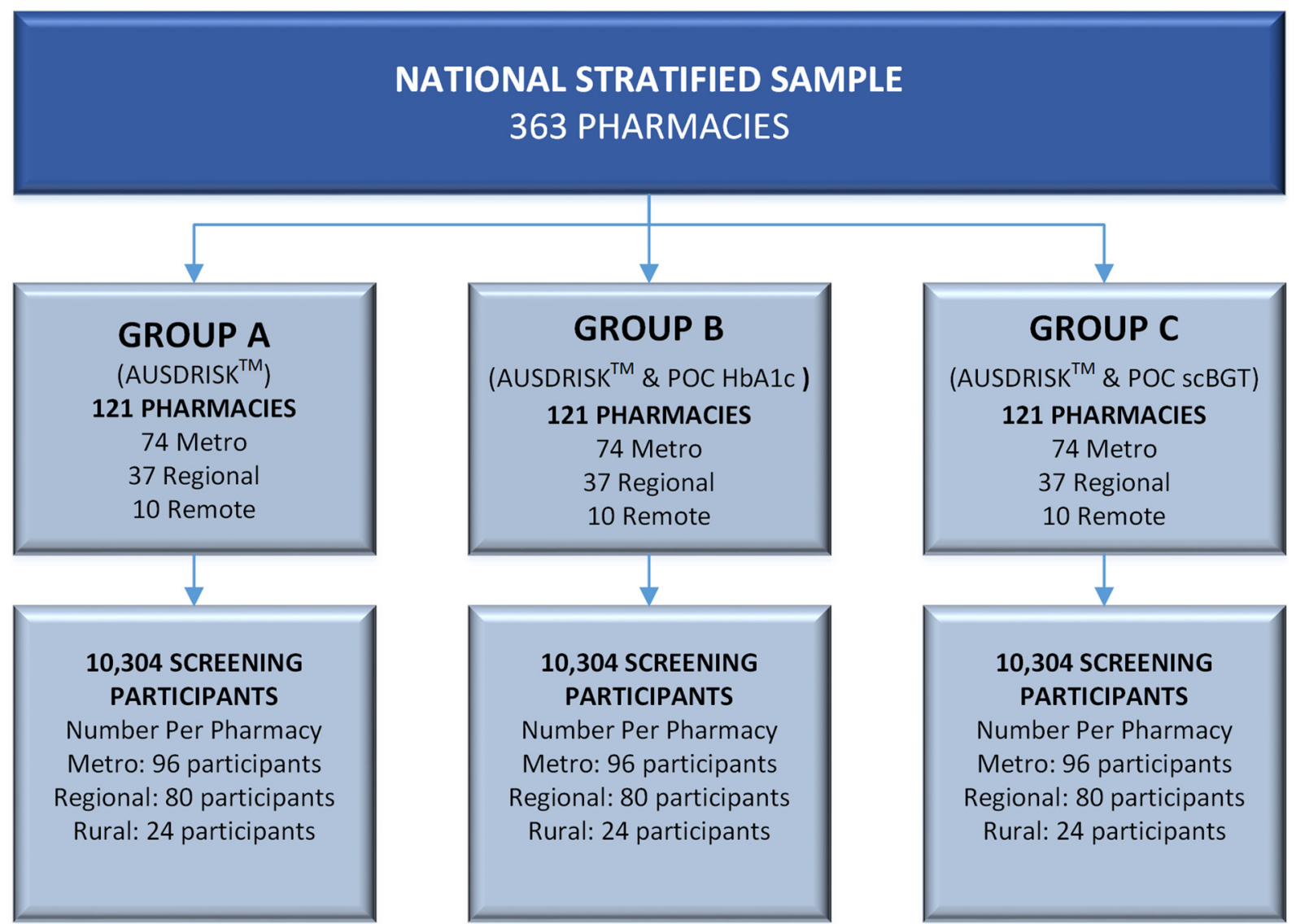

Figure 1 The Pharmacy Diabetes Screening Trial sample. HbA1c, glycated haemoglobin; POC, point of care; scBGT, small capillary blood glucose testing.

sample size calculation to account for age groups and gender separately. PASS $14^{34}$ will be used for sample size calculation.

A total sample size of 30912 screening participants (ie, 10304 per group) is required in order to: (1) detect a $30 \%$ relative improvement in the ratio of positive predictive values (PPVs) of the screening methods (with AUSDRISK PPV as baseline), at the 5\% significance level with $80 \%$ power; (2) account for the pharmacy clustering effect (ie, the assumed intraclass correlations (ICC) of 0.001 on the proportion scale produced a design effect of 1.1 that accounts for correlations among screening participants within same cluster); and (3) allow for an attrition rate, of those who withdraw or who are lost to follow-up, of up to $50 \%$. Given the lack of evidence for the true value of ICC, an estimated ICC from a cluster randomised controlled trial on screening for T2DM and a systematic review summarising ICC patterns from primary care research have been used as guideline for ICG selection. ${ }^{35} 36$

To achieve the target sample size of screening participants through 363 pharmacies and to account for the T2DM incidence range across the age strata, ${ }^{3}$ the recruitment target for each pharmacy will be 96 (metropolitan), 80 (regional) or 24 (remote) screening participants, stratified by gender and age categories (table 2 ). The sample size variation between metropolitan, regional and remote pharmacies reflects the fact that a 'proportional to residential location sampling strategy' will be adopted.

\section{Pharmacies}

To be eligible, a community pharmacy must:

- be approved to dispense Pharmaceutical Benefits Scheme medicines

- have a separate counselling room or private counselling area

Table 2 Screening participant recruitment quotas by gender, age and residential classification (remote, regional and metropolitan)

\begin{tabular}{llll}
\hline & \multicolumn{2}{l}{ Gender } & \\
\cline { 2 - 3 } Age groups (years) & Male & Female & Total \\
\hline $35-44$ & $3,10,12$ & $3,10,12$ & $6,20,24$ \\
\hline $45-54$ & $3,10,12$ & $3,10,12$ & $6,20,24$ \\
\hline $55-64$ & $3,10,12$ & $3,10,12$ & $6,20,24$ \\
\hline $65-74$ & $3,10,12$ & $3,10,12$ & $6,20,24$ \\
Total & $12,40,48$ & $12,40,48$ & $24,80,96$ \\
\hline
\end{tabular}

A total sample of 24,80 and 96 screening participants (stratified by age groups and gender) will be recruited from remote, regional and metropolitan pharmacies, respectively. 


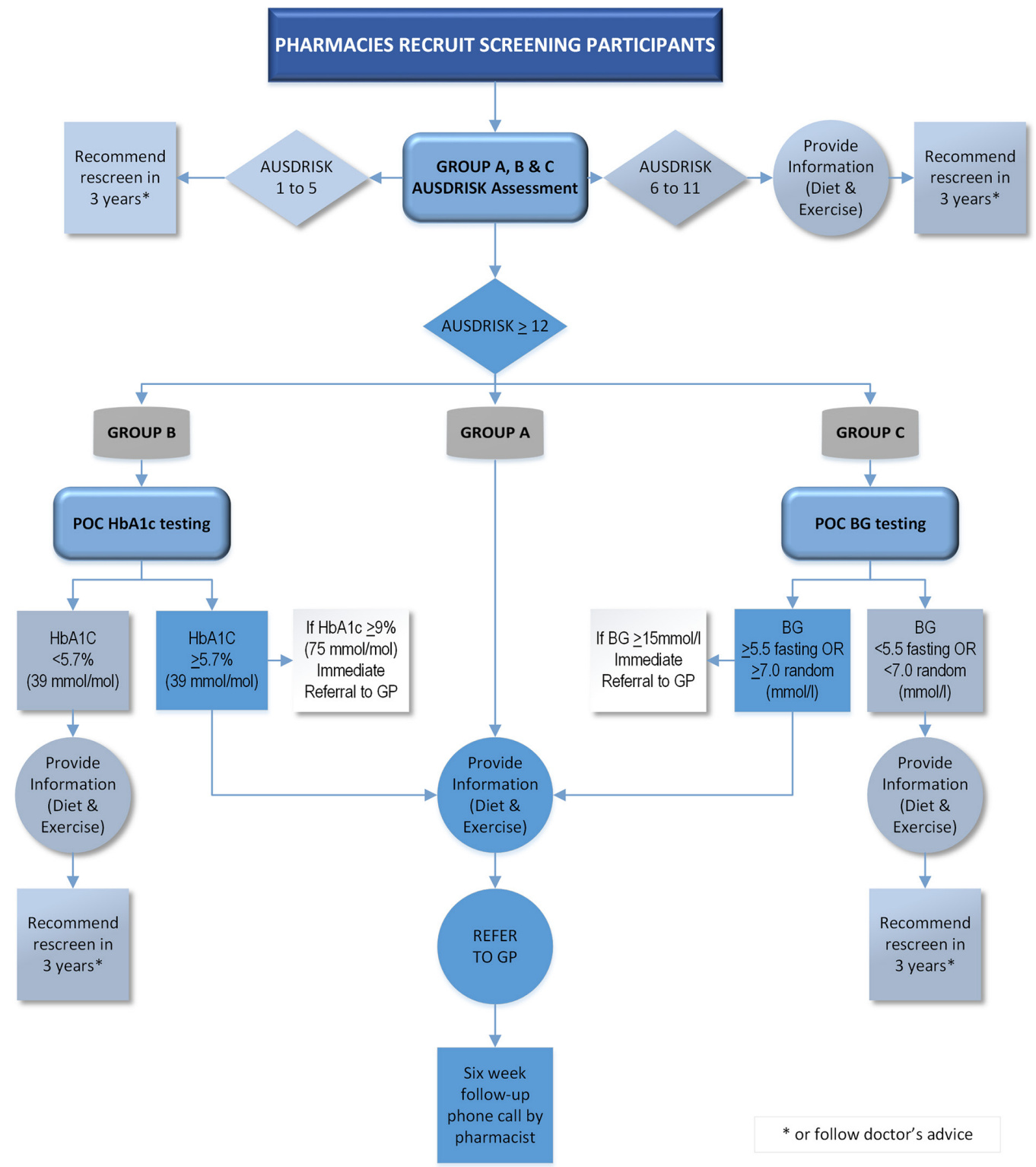

Figure 2 Clinical protocol. BG, blood glucose; GP, general practitioner; HbA1c, glycated haemoglobin; POC, point of care.

- be accredited by an approved Pharmacy Accreditation Program

- complete a baseline survey of existing screening diabetes services being conducted prior to commencing participation in the trial

- submit a trial implementation plan (via a proforma workbook).

All pharmacies in the selected geographical areas will be invited to participate and will be included providing they meet the eligibility criteria and give informed consent. Once they have provided written consent, they will be informed of their group allocation.

\section{Pharmacists}

Pharmacists who work in a selected pharmacy will be eligible to participate if they:

- are currently registered by the Australian Health Practitioner Regulation Agency
- satisfactorily complete a Continuing Professional Development (CPD)-accredited online training course and assessment

- agree to follow procedures outlined in the trial protocol

- demonstrate competence in POC testing using the device supplied for the trial (groups B and C).

The content for the CPD-accredited online training course will be developed by the project team and further developed for online delivery by the Guild Pharmacy Academy. This online training will consist of four modules: (1) trial overview; (2) about T2DM; (3) about screening; and (4) clinical protocol. Modules 1-3 will be the same for all groups, while module 4 is specific to each group and supported by standard operating procedures detailing each step of the clinical protocol (figure 2). The assessment component of the online training consists of 16 multiple-choice questions and requires an 
$80 \%$ pass mark for accreditation. Pharmacists will receive eight CPD points on accreditation and be eligible to claim additional CPD points for time spent conducting activities relating to the trial. The evaluation component of the online learning will include questions about their experience and overall level of satisfaction with the online training, the relevance of the activity to their practice and whether the course achieved the stated learning objectives.

To ensure consistency in waist measurements within and between pharmacies, a Seca 201 waist circumference tape measure will be provided to each pharmacy with instructions on best practice to take waist circumference measurements. ${ }^{37}$

Pharmacists in groups $\mathrm{B}$ and $\mathrm{C}$ will receive the Alere HbA1c Afinion Analyser AS100 or the Roche Accu-Chek Guide Blood Glucose System, respectively, for the duration of the trial. All participating pharmacies will receive in-pharmacy training sessions delivered by technical support representatives who will verify the pharmacist's proficiency in performing a POC test using a competency checklist, which is project specific and covers all aspects of obtaining a blood sample and use of the equipment. In addition, the trainer will make a video recording of the performance of the POC test to send to the project team for verification of competency. Pharmacists who successfully complete the Alere Afinion Analyser AS100 Analyser or Roche Accu-Chek Guide Blood Glucose System training programme may provide training to additional participating pharmacists in the trial if required. This also requires use of the competency checklist and provision of a video to the project team for review and approval of new trainees.

\section{Screening participants}

To be eligible screening participants must:

- be aged between 35 and 74 years

- not have been previously diagnosed with diabetes or pre-diabetes

- not have been screened for diabetes in the last 12 months

- not be enrolled in any lifestyle change programmes for T2DM

- not have a terminal illness or certain blood disorders (the latter includes severe haematological diseases, eg, thrombocytopaenia and leukaemia; shorter erythrocyte lifespan, eg, renal anaemia, chronic and haemolytic anaemia, acute blood loss and recent transfusion; haemoglobinopathy and red cell turnover disorders; and iron deficiency anaemia)

- be able to make independent decisions about their health.

Information about specific conditions will be provided as part of pharmacist training modules and protocols. Eligibility will be assessed on self-report by the prospective screening participant and based on the pharmacist being confident that they can make independent decisions about their health.

\section{Clinical protocol}

The clinical protocol for the PDST is summarised in figure 2 and illustrates the sequential steps of recruitment, AUSDRISK, POC test and referral to the GP.

Promotional material, including flyers and posters, will be provided to display in each participating pharmacy. The recruitment process involves discussing the PDST with customers and confirming eligibility in those who express an interest in being screened. All pharmacy staff, including pharmacists, pharmacy assistants and administrative staff, are actively encouraged to support the recruitment of screening participants. The expected duration of trial recruitment in the pharmacy is 4 months.

The screening appointment commences with the pharmacist opening a new record using tailored software hosted on GuildCare NG, an online cloud-based pharmacy recording platform, which will provide integrated guidance for recruitment and intervention delivery, automate key processes around patient referral and follow-up and enable the required data recording and collection for the trial. Eligible screening participants who are interested in participating will be given a participant information sheet to read and be asked to sign two consent forms-one to participate in the trial and one to give permission for the release of their Medicare data (data identifying government-reimbursed health services).

All screening participants will then be asked to complete the AUSDRISK and have their waist circumference measured by the pharmacist. GuildCare NG will calculate the AUSDRISK score and prompt the next step. Screening participants with an AUSDRISK score of $<6$ will receive information on rescreening in 3 years. Screening participants with an AUSDRISK score $\geq 6$ will receive risk reduction lifestyle information on diet and exercise. ${ }^{38}{ }^{39}$ All screening participants with an AUSDRISK score of $\geq 12$, in addition to diabetes risk reduction lifestyle information on diet and exercise, will also get a list of contacts for state-based diabetes prevention programmes where available.

The process for referred participants was adapted from RACGP guidelines ${ }^{40}$ through consultation with the PDST expert panel. In group A, screening participants with an AUSDRISK score of $\geq 12$ will be referred to the GP. In group $\mathrm{B}$, screening participants with an AUSDRISK of $\geq 12$ will receive an $\mathrm{HbA1c}$ POC test in the pharmacy using the Alere Afinion Analyser and those with an $\mathrm{HbAlc}>39 \mathrm{mmol} / \mathrm{mol}(5.7 \%)$ will be referred to the GP. In group $\mathrm{C}$, screening participants with an AUSDRISK $\geq 12$ will receive a scBGT POC test in the pharmacy using the Accu-Chek Guide and those with either a fasting blood glucose of $\geq 5.5 \mathrm{mmol} / \mathrm{L}$ (or a random blood glucose of $\geq 7.0 \mathrm{mmol} / \mathrm{L}$ ) will be referred to the GP. For those who qualify for GP referral based on the clinical protocol (figure 2), a referral letter outlining their AUSDRISK and POC results will be automatically generated and given to the screening participant to take to their GP. With the screening participant's consent, the pharmacist will also fax the referral letter to the nominated GP. 
In the event of a very high $\mathrm{HbAlc}$ result (ie, $\geq 75 \mathrm{mmol} /$ mol $(9 \%)$ ) or blood glucose result $(\geq 15 \mathrm{mmol} / \mathrm{L})$ during the screening appointment, GuildCare NG will trigger a notification for the pharmacist to arrange an immediate appointment with the screening participant's nominated GP.

All referred screening participants will be contacted by the pharmacist 6 weeks after the screening appointment to check on the status of their recommended follow-up with the GP and to motivate referred patients who have not yet made an appointment with their GP. Non-referred participants will receive a copy of their screening report printed from GuildCare NG outlining their risk factors and their AUSDRISK results.

\section{PDST data collection and reporting}

The PDST clinical protocol data collection system will be purpose designed to operate on the newly developed software platform GuildCare NG, which will be provided to participating pharmacies with group specific guides to support access and familiarisation. Data reports from GuildCare NG will be uploaded to Power BI to enable monitoring of individual pharmacy recruitment and allow early intervention where pharmacies may require assistance with recruitment. Regular data files will also be supplied to the project team for data analysis as well as follow-up and determination of screening outcomes.

Eligible participating pharmacies will receive per-patient payments (via a payment file in GuildCare NG) for each component of the screening service they complete as follows: AUSDRISK $\$ 10.00$; POC test $\$ 10.50$; and Referral \$11.00. In addition, at the end of service provision, a bonus of $\$ 750.00$ will be provided to the pharmacies as an incentive, payable on the completeness of the data collected according to the protocol.

\section{Clinical outcomes}

The screening participants' referral outcomes will be classified as 'true positives' or 'false positives', based on whether they subsequently obtain a T2DM diagnosis, respectively. The PPVs of the assessed T2DM screening and testing methods will also be calculated to allow comparison with other screening and testing modalities, which can be undertaken within the scope of health economic evaluation. However, the calculation of 'true negatives', 'false negatives' and 'negative predictive values' is outside the scope of this project.

The outcomes of the GP referral will be determined using a stepwise approach involving triangulation of the following data sources: (1) contact with the GP practice and/or pathology laboratory; (2) a follow-up survey of screening participants who were referred to their GP at 3 months postscreening; and (3) use of Medicare and National Diabetes Support Scheme (NDSS) data to identify items relating to diagnostic testing for diabetes. A diagnosis will be considered positive if confirmed by a GP, pathology lab, Medicare or NDSS data.

\section{Data analyses}

The clinical protocol for the PDST (figure 2) will adhere to 'intention-to-treat' (ITT) principles and follow the Consolidated Standards of Reporting Trials (CONSORT) Statement guidelines in reporting the findings $^{41}$ to include non-attenders as well as those lost to follow-up.

Clinical outcome analyses will be based on all screening participants who meet the eligibility criteria and provide written consent. The null hypothesis of no difference between groups will be tested against alternative hypothesis that either group B or group $\mathrm{C}$ will show significant differences in clinical and economic outcome measures. Since we are only interested in two prespecified comparisons in this three-armed trial (ie, group A vs group B; group A vs group C) and we have a priori expectation about direction of the alternative hypothesis, no adjustment is made for multiple comparison. ${ }^{42}$ While the direction of alternative hypotheses are prespecified, one-tailed tests are avoided, and all statistical tests are two-tailed tests. ${ }^{44}$

For the primary and all secondary clinical outcomes, the CMH test will be used to compare the differences in proportions of true T2DM detected between groups. This will be done by performing $\mathrm{CMH}$ tests for group A versus group $\mathrm{B}$ and group A versus group $\mathrm{C}$ comparisons. $\mathrm{CMH}$ is an efficient approach to compare clustered binary outcomes. ${ }^{45}$ The CMH test takes account of the cluster randomisation by considering each pharmacy as a strata and allows for variation between the strata in the underlying rates. The common OR and its $95 \%$ CI will also be reported as well as the results of the Breslow-Day test for homogeneity of the ORs across the strata. If there is significant heterogeneity in the ORs, the screening groups will also be compared using $\chi^{2}$ tests using separate subset analyses. In further supportive analyses, generalised linear mixed models (GLMMs) with binary outcome and logit link will be used to simultaneously compare the proportion of newly diagnosed T2DM in group A vs group B and group A vs group C. Generalised estimating equations approach with working correlation structure and robust variance estimator will be used for this purpose. ${ }^{46}$ GLMMs will account for pharmacy clustering effects, baseline measurements and explore possible interactions between baseline factors and screening methods.

The baseline independent variables of interest are gender, age groups and waist circumference of screening participants as well as socioeconomic, location and remoteness indices of pharmacies (ie, metro, regional and remote). Any baseline measure with estimated risk greater than $20 \%$ according to bivariate or estimation using GLMMs will be adjusted for in the multivariate models. In additional analyses, random effect logistic regression will be used to estimate ICC on logistic scale.

Subgroup analyses will involve testing for associations with the primary outcomes for the following additional independent variables: baseline pharmacy characteristics, socioeconomic profile of the community in which the 
pharmacy is located, different threshold of AUSDRISK, and different thresholds for blood tests, nature and extent of counselling after screening, and successful completion of the 6-week follow-up. Other intended substudies include analyses to determine factors associated with uptake of referral to GP and subsequent diagnostic testing, as well as factors associated with pharmacy performance during the trial (recruitment rates and intervention fidelity).

Missing values will be scrutinised to check for non-random distribution and analyses that use baseline data will be executed twice: once using observed data, and once using the multiple imputation (MI) method. ${ }^{47}$ Participant characteristics and other important measures, such as AUSDRISK results, will be compared in complete and missing data in order to detect systematic patterns in missing data. Sensitivity analysis will be conducted to evaluate random assumptions for missing follow-up data, which will be imputed assuming missing not at random (MNAR) in all three arms. Robustness of the findings under different MNAR assumptions will be examined by imputing missing data using the MI approach. The sensitivity analyses will account for all randomised participants. The first set of MIs assume missing at random, while the other sets of MIs will be implemented under MNAR assumptions. In MNAR scenarios imputation of missing outcomes will be associated with higher risk (ie, higher diabetes proportion) in group B and group C compared with group A. All reasonable scenarios will be considered in the sensitivity analyses. The robustness of the conclusion with regard to the treatment difference will be evaluated across these scenarios, and a range of profiles for undiagnosed diabetes due to missing follow-up referral will be examined for their impact on the study conclusion. ${ }^{48}$

\section{PDST evaluation}

A logic model (figure 3) summarises the steps in the PDST programme development, implementation and projected outcomes. This logic will be used to frame our approach to evaluating: (1) whether the PDST was implemented as intended (fidelity and reach); (2) the way in which study outcomes were achieved or not achieved; and (3) the influence of situational factors on the results obtained. Baseline survey data, screening data and pharmacist interview transcripts will be used to assess these issues.

\section{Baseline evaluation}

At the beginning of the trial, participating pharmacies will be required to complete a baseline survey that includes questions on pharmacy characteristics, existing professional services and strategies used for implementing these services. This information will be used to inform the comparability of trial arms and health economic analysis.

\section{Screening data}

All data entered into GuildCare NG during screening by pharmacists will be extracted centrally to enable

\begin{tabular}{|c|c|c|c|c|}
\hline INPUTS & ACTIVITIES & OUTPUTS & SHORT TERM OUTCOMES & LONG TERM OUTCOMES \\
\hline $\begin{array}{l}\text { Financial } \\
\text { Grant from Tranche } 1^{\text {st }}-6^{\text {th }} \text { CPA } \\
\text { Pharmacy trials program } \\
\text { To cover costs of trial administration, } \\
\text { remuneration to pharmacies, } \\
\text { development of IT software, cost of } \\
\text { testing equipment and consumables, } \\
\text { evaluation } \\
\text { Users and Stakeholders } \\
\text { Consumers (patients) } \\
\text { Community pharmacy pharmacists } \\
\text { Community pharmacy staff } \\
\text { GPs and their practice staff } \\
\text { Australian government } \\
\text { Technology Components } \\
\text { Guildlink }{ }^{\text {TM }} \text { electronic recording } \\
\text { software for service documentation } \\
\text { Training } \\
\text { Online CPD accredited training } \\
\text { program developed for participating } \\
\text { pharmacists } \\
\text { Other Resources } \\
\text { Material resources (e.g. Promotional } \\
\text { material (signage, flyers etc., health } \\
\text { information handouts for screening } \\
\text { participants), POC testing equipment } \\
\text { (BGL and HbA1c analysers plus test } \\
\text { strips, cartridges and other associated } \\
\text { consumables)) }\end{array}$ & $\begin{array}{l}\text { 1. Preliminary } \\
\text { Expert panel convened to provide } \\
\text { advice on trial implementation; } \\
\text { and how to support engagement } \\
\text { with GPs, PHNs, recruit, retain } \\
\text { participants, community } \\
\text { pharmacies and other relevant } \\
\text { health professionals } \\
\text { Recruit Pharmacies } \\
\text { 2. Implementation of cRCT } \\
\text { Pharmacist Intervention } \\
\text { - Initial training } \\
\text { - Implementation planning } \\
\text { - Recruitment and screening of } \\
\text { eligible patients } \\
\text { Referral of screened } \\
\text { participants at elevated risk } \\
\text { of diabetes to GP according } \\
\text { to clinical protocol } \\
6 \text { week follow up of referred } \\
\text { participants } \\
\text { Regular newsletter on trial } \\
\text { progress }\end{array}$ & $\begin{array}{l}\text { - Improved rates of } \\
\text { detection of people at } \\
\text { elevated risk of diabetes } \\
\text { (and subsequent } \\
\text { diagnosis) } \\
\text { - Improved rates of } \\
\text { detection of people with } \\
\text { pre-diabetes } \\
\text { - Improved awareness of } \\
\text { lifestyle modification to } \\
\text { reduce diabetes risk } \\
\text { Improved uptake of } \\
\text { lifestyle risk reduction } \\
\text { programs } \\
\text { Improved collaboration } \\
\text { and continuity of care } \\
\text { between pharmacist and } \\
\text { GPs }\end{array}$ & $\begin{array}{l}\text { PDST Outcomes } \\
\text { Which of the three approaches to } \\
\text { screening and referral for diabetes } \\
\text { risk was the most clinically and cost } \\
\text { effective? } \\
\text { Primary Outcomes } \\
\text { - \% of newly diagnosed T2DM, } \\
\quad \text { according to the criteria of the } \\
\text { Australian Diabetes Association: } \\
\text { - } \% \text { diagnosed with pre-diabetes } \\
\text { ICERs } \\
\text { Secondary Outcomes } \\
\text { - } \% \text { who have been referred to the } \\
\text { GP who take up the referral to the } \\
\quad \text { GP } \\
\text { Was the screening protocol } \\
\text { implemented as intended? } \\
\text { Internal Fidelity } \\
\text { Did the pharmacists screen clients } \\
\text { according to protocol? } \\
\text { Reach } \\
\text { Were they able to screen the target } \\
\text { numbers and what were the } \\
\text { characteristics of those screened? }\end{array}$ & $\begin{array}{l}\text { - } \begin{array}{l}\text { Reduced incidence of } \\
\text { type } 2 \text { diabetes }\end{array} \\
\text { - Guide health policy to } \\
\text { efficient us of primary } \\
\text { care health resources }\end{array}$ \\
\hline
\end{tabular}

Figure 3 The Pharmacy Diabetes Screening Trial (PDST) logic model. BGL, blood glucose level; CPA, Community Pharmacy Agreement; cRCT, clustered randomised controlled trial; GP, general practitioner; HbA1c, glycated haemoglobin; ICERs, incremental cost-effectiveness ratios; PHN, Primary Health Network; POC, point of care; T2DM, type 2 diabetes mellitus. 
quantitative elements of process evaluation around the screening process.

\section{Midtrial interviews}

Two months after completion of online training, a mix of pharmacists from high-performing and low-performing pharmacies in all three trial arms will be invited to provide feedback individually or as part of a focus group involving participants from their trial arm. The purpose of obtaining feedback will be to identify challenges, opportunities, barriers and facilitators to service implementation. This will be used by researchers to conduct a midtrial review of pharmacy support and processes, with adaptation where feasible and dissemination of effective implementation strategies identified.

\section{End-of-trial interviews}

At the end of the trial, a number of surveys, telephone questionnaires/interviews and focus groups will be conducted as follows:

1. A purposive sample of $10 \%$ of participating pharmacists, representing a mix of high recruitment and low recruitment, will be invited to participate in semistructured interviews and focus groups to gain feedback on service implementation in the pharmacy, including barriers and facilitators to implementing the trial in the pharmacy, perceptions of sustainability and level of engagement by locals GP in the referral process.

2. All referred participants will receive an online survey to determine the outcome of the referral, as well as their level of satisfaction with the service.

3. A random sample of $20 \%$ of non-referred screening participants will be surveyed to determine their overall experience and level of satisfaction with the service.

4. A random sample of $10 \%$ of GPs who had patients referred to them during the trial will be invited to respond to a telephone questionnaire to determine their overall experience and level of satisfaction with the service.

All surveys will be created using recognised survey software, for example, Research Electronic Data Capture $(\text { REDCap })^{49}$, a secure web-based application designed to support data capture for research studies hosted at the University of Sydney. REDCap provides an intuitive interface for validated data entry, audit trails for tracking data manipulation and export procedures, automated export procedures for seamless data downloads to common statistical packages and procedures for importing data from external sources.

Qualitative interviews will be conducted with the aid of the ethics approved semistructured interview guide, which will comprise open-ended questions that prompt discussion on a wide range of topics including their experience with the PDST, consumer feedback, interaction with GPs and impact of the trial in their pharmacy. Qualitative interviews will be conducted by members of the research team, and a maximum variation sample of PDST pharmacists, reflecting a range of location, capacity and indicators of trial success, will be invited to participate in either individual interviews or focus groups. The interview process will continue until data saturation is achieved. Interviews will be audiotaped and transcribed verbatim, and a thematic analysis performed using standard qualitative approaches. NVivo software will be used as a tool in identifying, labelling and organising themes.

\section{Impact evaluation}

This will be determined by testing the clinical hypotheses of the trial as previously outlined in sections 'Clinical Protocol' and 'Clinical Outcomes'.

\section{Economic evaluation}

A 'cost-effectiveness analysis' will be conducted from a health service funder perspective that includes costs borne by the pharmacists, the government (Medicare Benefits Scheme (MBS) items) and the PDST administration. Costs borne by the patients (eg, copayments charged by GPs) are not included in the analysis.

The core hypothesis for the economic analysis is that addition of either the HbAlc POC test or the scBGT POC test to AUSDRISK screening followed by a referral to GP, if appropriate, is cost-effective in comparison with AUSDRISK screening alone. The 'net cost per confirmed new T2DM diagnosis' for each group will be estimated and compared. We will assess whether the average cost of the new T2DM case in either of the POC intervention groups is lower or higher than the average cost of the new T2DM case in the AUSDRISK alone group.

There is likely to be a difference in average costs per new T2DM diagnosis across the sites in the same cluster and across the clusters from the metropolitan, regional and remote settings. This is due to the difference in the numbers of the recruited patients (that can defy the predetermined sample size), local unit prices, staff mix and established local practices. The source of variation (if observed) as well as the cost drivers will be investigated with a generalised linear model using one of the standard statistical software packages (SPSS V.24 or STATA V.14).

In addition to preparation of average cost-effectiveness ratios per group, incremental cost-effectiveness ratios (ICERs) will also be prepared that allow fuller analysis of incremental costs across various comparator combinations in various settings.

Depending on the trial-based outcomes, the cost-effectiveness analysis conducted alongside the trial (time horizon determined according to average time from screening to the confirmation of T2DM status based on the lab test results), will be complemented by a modelled economic evaluation with an extended time horizon (eg, the expected lifetime for each age subgroup of the eligible population). The modelled economic evaluation would translate the benefits of early diagnosis of T2DM and the associated prevention/delay of the T2DM complications into final health benefits, assessed in life-years saved 
and quality-adjusted life-years (QALYs). The modelled economic evaluation will depict the processes and clinical management algorithms beyond the point of T2DM diagnosis (ie, 'within trial' observation period) and will include the costs of treatment, regular laboratory investigations, treatment of adverse events, hospital admissions and other relevant costs. The long-term improved outcomes are hypothesised to translate in a cost reduction and offset the increased costs associated with adding pharmacy-based screening for T2DM to the GP-based diagnosis services. The modelled economic evaluation will draw on the published results of long-term observational studies in order to derive assumptions for the decision analytic modelling and to populate the model. An appropriate discount rate will be applied to both costs and benefits incurred over the longer time horizon.

In the cost analysis, both fixed and variable costs will be assessed. The former will include durable items of equipment, time involved in the development of materials (including the online materials) used in the preparation for and administration of the service, staff training and IT input into developing a software platform for collection of patient personal and medical data. The variable costs also include the promotional resources kit, POC kit, disposable materials, blanks of the questionnaires and so on. The sources for the cost data include the screening participants' records entered by the pharmacies into GuildCare NG, MBS data and pharmacists' reports on the time spent on screening/testing the screening participants. The reports can be complemented by the direct observations at randomly selected pharmacies.

The variability in effect sizes and cost differences generates a degree of uncertainty, while any uncertainty in these parameters translates into uncertainty in the ICER estimate. However, generating a $95 \%$ CI for an ICER is problematic as the ratio of the two distributions does not necessarily have a finite mean or, therefore, a finite variance. ${ }^{50}$ The uncertainty about results of economic evaluation, that is, around the ICER estimate, will be explored using a non-parametric bootstrapping method with at least 1000 resampling draws. Results will be plotted on the cost-effectiveness plane to illustrate the uncertainty cloud and also on an acceptability curve to illustrate the certainty of a cost-effective result for a chosen threshold (eg, 90\% confidence levels of all ICER results are $<\$ 50$ 000 per QALY). The CI is estimated by using percentile interval method as a quasi-CI, since the non-parametric bootstrap method does not produce a true statistical inference for a proportion. Although the accuracy of estimation using the percentile interval could be potentially compromised, the method is commonly used in economic evaluation. This reflects its ease of use, acceptable rigour and advantages in communicating research findings to a broader audience, including policy makers and clinicians.

Interpretation of the results would depend on the position of the distribution of bootstrapped ICERs on the cost-effectiveness plane. If the AUSDRISK alone assessment is associated with a higher rate of false positive cases, then it may attract higher costs. ${ }^{50}$ For example, for an intervention that is more effective and more costly than the comparator, the positive decision to invest would depend on the decision maker's maximum willingness to pay per additional unit of the outcome, that is, per additional case of new T2DM diagnosis. Alternatively, if 'dominance' is demonstrated (ie, POC intervention arms cheaper than AUSRISK screening alone for the same or better health outcomes), then efficiency is clearly evident.

Brief mention was made above of financial appraisal being specified alongside economic appraisal. This is important to clarify in the protocol as it impacts on how the economic analysis is conducted. It is not uncommon, for example, that where costs occur in all arms of a trial, for these costs to be excluded from the economic analysis as not influencing the ICERs. This may become problematic later on, however, if decision makers want to know the full budget required to implement the initiative evaluated. Financial appraisal and full pathway costing will be undertaken as part of this trial. Where assumptions for the identification, measurement and valuation of economic costs differ to financial costs, these differences will be specified.

The results of process, impact and economic evaluation will determine the most clinically effective and cost-effective screening model as well as the feasibility of its future translation and sustainability in community pharmacy.

\section{Ethics and dissemination}

The protocol has been approved by the Human Research Ethics Committees at University of Sydney and Deakin University. Results will be available on the Sixth Community Pharmacy Agreement (6CPA) website and will be published in peer-reviewed journals.

Approval has been granted from the Australian Government Department of Human Resources to obtain screening participants' MBS items relating to claimed fees for health service provision during the trial.

\section{DISCUSSION}

A comparative analysis will establish whether an elevated AUSDRISK risk score combined with an elevated POC test result (groups B and C) is more clinically effective and cost-effective than the AUSDRISK elevated risk score alone at detecting T2DM in the community.

It has previously been shown that pharmacy-based screening for T2DM, using a combination of a risk assessment tool followed by a scBGT POC test, was associated with a statistically significant increase in the proportion of newly diagnosed T2DM cases, although the proportion of false positive results (based on an ITT analysis) was high in both the intervention and the comparator arms. ${ }^{24}$ The PDST aims to expand this study to a much larger scale, as well as refining the protocol by using the AUSDRISK diabetes risk assessment tool, which is currently the benchmark for use in general practice throughout 
Australia, as well as including a group that uses HbA1c POC test for those with AUSDRISK $\geq 12$, compared with scBGT POC test for those with AUSDRISK $\geq 12$ or AUSDRISK $\geq 12$ alone.

The protocol for this pharmacy-based screening trial is centred on correctly identifying diabetes risk factors and referring those who meet the criteria to a GP for further testing and possible diagnosis. The thresholds for HbAlc, fasting and random blood glucose used for identifying people at risk in this trial are set lower than those used for diagnostic tests. ${ }^{40}$ This is to minimise false negatives, that is, those with the disease who do not meet the criteria for referral to the GP, which by default will result in more false positives, that is, those without the disease that meet the criteria for referral to the GP. These parameters have important health status and cost implications, which are useful for policy analysis. A high false positive rate, for example, adds substantial follow-up/confirmation costs to the screening pathway, as well as unnecessary patient anxiety. A high false negative rate, however, would mean patients with diabetes are being missed by the screening algorithm that may have serious adverse consequences.

The overall expected attrition rate of $50 \%$ may include disproportionate withdrawal from particular groups. To reduce the impact of any potential bias, the ITT analysis will include all screening participants in the final analysis, regardless of non-compliance or withdrawal. ITT is also appropriate and preferred from an economic evaluation perspective for allocative efficiency but would not exclude 'per protocol' analysis to examine technical efficiency issues related to intervention design and degree of exposure. Medicare data will be used, where possible, to estimate the outcomes for those lost to follow-up.

Pharmacies that represent a wide range of local demographics and regions across Australia will be selected to participate in the PDST. The representative and random nature of pharmacy recruitment will also inform the potential real-world effectiveness of each intervention at a population level. However, while every effort has been made to provide standardised approaches and clear documentation, as well as appropriate answer fields to minimise errors in participant responses, we acknowledge that some data obtained from pharmacists may be inaccurate or incomplete. In addition, recruitment of screening participants may not have the expected uptake, and we may be unable to verify whether a diagnosis of T2DM or pre-diabetes was made for a small proportion of referred screening participants whose diabetes testing does not trigger NDSS registration, and where follow-up with the patient, pathology lab and GP cannot be achieved or otherwise fails to confirm an outcome.

As the prevalence of T2DM continues to rise, there is a need for novel programmes that provide effective screening for undiagnosed T2DM that can reach into all areas of Australia. The PDST addresses goal 2 of the Australian National Diabetes Strategy, ${ }^{19}$ that is, to promote awareness and early detection in that it enables opportunistic screening of individuals during routine encounters with the community pharmacy in a previously undiagnosed population. The clinical analyses will evaluate which service has the most reliability and validity, while minimising discomfort and harm. The economic analyses will detail the costs of providing each service protocol and measure against cost impacts to the healthcare system. The process evaluation will provide information on how the trial service fits into the day-to-day operations of the pharmacy and its sustainability as well as satisfaction with the service from participants and GPs. A screening programme that is clinically effective, cost-effective and sustainable will result in many benefits and reduce the burden on the individual, the community and the health system.

\section{Author affiliations}

${ }^{1}$ Faculty of Pharmacy, University of Sydney, Sydney, New South Wales, Australia ${ }^{2}$ Deakin Health Economics, Centre for Population Research, Faculty of Health, Deakin University, Burwood, Victoria, Australia

${ }^{3}$ Biostatistics unit, Faculty of Health, Deakin University, Geelong, Victoria, Australia ${ }^{4}$ School of Medicine, Faculty of Health, Centre for Population Health Research, Deakin University, Burwood, Melbourne, Victoria, Australia

${ }^{5}$ Deakin University School of MedicineWarrnambool Campus, Deakin University, Warnambool, Victoria, Australia

Acknowledgements This trial has been developed in partnership with the Pharmacy Guild of Australia, University of Sydney and Deakin University. We wish to acknowledge the contribution of the Pharmacy Guild of Australia to the implementation of this trial.

Contributors IK and KM developed the overall design of the trial and edited the manuscript. RC and STFS designed the components for the economic analysis. VLV and MM created computerised algorithms to generate the pharmacy randomised sampling strategy and wrote the sections on statistical analysis, sample size and sampling of pharmacies. BM prepared the manuscript. PT, FW and BM wrote the training materials, prepared the ethics applications and the tables and figures.

Funding The Pharmacy Diabetes Screening Trial is funded by the Australian Government Department of Health as part of the Sixth Community Pharmacy Agreement.

\section{Competing interests None declared.}

Ethics approval Human Ethics Committee of the University of Sydney, Deakin University Human Ethics Committee.

Provenance and peer review Not commissioned; externally peer reviewed.

Open Access This is an Open Access article distributed in accordance with the Creative Commons Attribution Non Commercial (CC BY-NC 4.0) license, which permits others to distribute, remix, adapt, build upon this work non-commercially, and license their derivative works on different terms, provided the original work is properly cited and the use is non-commercial. See: http://creativecommons.org/ licenses/by-nc/4.0/

(C) Article author(s) (or their employer(s) unless otherwise stated in the text of the article) 2017. All rights reserved. No commercial use is permitted unless otherwise expressly granted.

\section{REFERENCES}

1. Kahn SE, Cooper ME, Del Prato S. Pathophysiology and treatment of type 2 diabetes: perspectives on the past, present, and future. Lancet 2014;383:1068-83.

2. Australian Institute of Health and Welfare. Cardiovascular disease, diabetes and chronic kidney disease: Australian facts: prevalence and incidence. in Cardiovascular, diabetes and chronic kidney disease. Series no 2. Canberra, Australia: Australian Institute of Health and Welfare, 2014.

3. Australian Bureau of Statistics. National Health Survey Australia 2014-2015. Canberra, Australia: Australian Bureau of Statistics. 
4. Diabetes Australia. Diabetes in Australia. 2017 https://www. diabetesaustralia.com.au/diabetes-in-australia (accessed $15 \mathrm{Apr}$ 2017).

5. International Diabetes Federation. The IDF Diabetes Atlas. 7th Edition, 2015. http://www.diabetesatlas.org/resources/2015-atlas. html. (accessed 15 Apr 2017).

6. Baker IDI Heart and Diabetes Institute. Diabetes: the silent pandemic and its impact on Australia. Melbourne, Australia: Baker IDI in partnership with Diabetes Australia and Juvenile Diabetes Research Foundation, 2012.

7. Magliano DJ, Barr EL, Zimmet PZ, et al. Glucose indices, health behaviors, and incidence of diabetes in Australia: the Australian Diabetes, Obesity and Lifestyle Study. Diabetes Care 2008;31:267-72.

8. Barr EL, Zimmet PZ, Welborn TA, et al. Risk of cardiovascular and allcause mortality in individuals with diabetes mellitus, impaired fasting glucose, and impaired glucose tolerance: the Australian Diabetes, Obesity, and Lifestyle Study (AusDiab). Circulation 2007;116:151-7.

9. Dunstan DW, Zimmet PZ, Welborn TA, et al. The Australian Diabetes, Obesity and Lifestyle Study (AusDiab)--methods and response rates. Diabetes Res Clin Pract 2002;57:119-29.

10. Dunstan DW, Zimmet PZ, Welborn TA, et al. The rising prevalence of diabetes and impaired glucose tolerance: the Australian Diabetes, Obesity and Lifestyle Study. Diabetes Care 2002;25:829-34.

11. National Health and Medical Research Council. National evidence based guidelines for the management of type 2 diabetes mellitus. Part 3 Case detection and diagnosis of type 2 diabetes. Canberra, Australia: National Health and Medical Research Council, 2001.

12. Colagiuri S, Davies D, Girgis S, et al. National evidence based guidelines for case detection and diagnosis of type 2 diabetes. Canberra, Australia: Diabetes Australia and the NHMRC, 2009.

13. Norris SL, Zhang X, Avenell A, et al. Long-term non-pharmacological weight loss interventions for adults with type 2 diabetes mellitus. Sao Paulo Med J 2016;134:184.

14. Norris SL, Zhang X, Avenell A, et al. Long-term non-pharmacological weight loss interventions for adults with prediabetes. Cochrane Database Syst Rev 2005;2:CD005270.

15. Tuomilehto J, Lindstrom J, Eriksson JG, et al. Finnish Diabetes Prevention Study Group. Prevention of type 2 diabetes mellitus by changes in lifestyle among subjects with impaired glucose tolerance. N Engl J Med 2001;344:1343-50.

16. Chen L, Magliano DJ, Balkau B, et al. AUSDRISK: an Australian Type 2 Diabetes Risk Assessment Tool based on demographic, lifestyle and simple anthropometric measures. Med J Aust 2010;192:197-202.

17. Australian Bureau Statistics. Australian Aboriginal and Torres Strait Islander health survey: updated results, 2012-13 Australian Bureau Statistics 2012-2013. Canberra, Australia: Australian Bureau Statistics.

18. Australian Institute of Health and Welfare. How many Australians have diabetes? Canberra, Australia: Australian Institute of Health and Welfare, 2014.

19. Australian Government Department of Health. Australian National Diabetes Strategy 2016 -2020. Canberra: Australian Government Department of Health: Commonwealth of Australia.

20. Willis A, Rivers P, Gray LJ, et al. The effectiveness of screening for diabetes and cardiovascular disease risk factors in a community pharmacy setting. PLoS One 2014;9:e91157.

21. The Pharmacy Guild of Australia. Community Pharmacy - a trusted public-private partnership delivering accessible high quality healthcare for all Australians. 2014 https://www.guild.org.au/ data/ assets/pdf_file/0010/6121/pgoa-submission-to-the-competitionpolicy-review-june-2014_573492_3.pdf (accessed 15 Apr 2017).

22. Menzies Centre for Health Policy. The Menzies-Nous Australian Health Survey 2012: Univeristy of Sydnen, 2012. http://www. nousgroup.com/images/news_attachments/Menzies-Nous Australian_Health_Survey_Report_2012.pdf. (accessed 17 Apr 2017).

23. Sookaneknun P, Saramunee K, Rattarom R, et al. Economic analysis of the diabetes and hypertension screening collaboration between community pharmacies and a Thai government primary care unit. Prim Care Diabetes 2010;4:155-64.

24. Krass I, Mitchell B, Clarke P, et al. Pharmacy diabetes care program: analysis of two screening methods for undiagnosed type 2 diabetes in Australian community pharmacy. Diabetes Res Clin Pract 2007;75:339-47.

25. Mc Namara K, Peterson G, Hughes J, et al. Cardiovascular disease risk assessment in Australian community pharmacy. Heart Lung Circ. In Press.;2016.
26. Malo JA, Versace VL, Janus ED, et al. Evaluation of AUSDRISK as a screening tool for lifestyle modification programs: international implications for policy and cost-effectiveness. BMJ Open Diabetes Res Care 2015;3:e000125.

27. Australian Bureau of Statistics. Australian Social Trends. 2008 http://www.abs.gov.au/AUSSTATS/abs@.nsf/Lookup/4102. 0Chapter3002008

28. Quality Care Pharmacy Program. http://www.qcpp.com (accessed 15 Apr 2017).

29. Australian Bureau of Statistics. Australian Statistical Geography Standard (ASGS). 2011. Volume 3 - Non ABS Structures. 2011 http://www.abs.gov.au/AUSSTATS/abs@.nsf/DetailsPage/1270.0.55. 003July\%202011? OpenDocument

30. Australian Bureau of Statistics. Socio-Economic Indexes for Areas. 2013 http://www.abs.gov.au/websitedbs/censushome.nsf/home/ seifa (accessed 17 Apr 2017).

31. Australian Bureau of Statistics. Australian Statistical Geography Standard (ASGS). 2011. Volume 5 - Remoteness Areas. 2011 http:// www.abs.gov.au/websitedbs/d3310114.nsf/home/remoteness+ structure (accessed 17 Apr 2017)

32. Dunstan D, Zimmet P, Welborn T, et al. Diabesity and associated disorders in Australia, 2000: The accelerating epidemic: The Australian Diabetes, Obesity and Lifestyle Study (AusDiab: International Diabetes Institute, 2001.

33. Nam J. Sample size determination for case-control studies and the comparison of stratified and unstratified analyses. Biometrics 1992;48:389-95.

34. PASS 14 Power analysis and sample size software. Kaysville, Utah, USA: NCSS, LLC. https://www.ncss.com/software/pass/. 2015.

35. Simmons RK, Echouffo-Tcheugui JB, Sharp SJ, et al. Screening for type 2 diabetes and population mortality over 10 years (ADDITION-Cambridge): a cluster-randomised controlled trial. Lancet 2012;380:1741-8.

36. Adams G, Gulliford MC, Ukoumunne OC, et al. Patterns of intracluster correlation from primary care research to inform study design and analysis. J Clin Epidemiol 2004;57:785-94.

37. World Health Organisation. Waist circumference and waist-hip ratio. Report of a WHO Expert Consultation. Geneva, 2008. http://apps. who.int/iris/bitstream/10665/44583/1/9789241501491_eng.pdf. (accessed 15 Apr 2017).

38. Australian Government Department of Health. Healthy eating for adults. https://www.eatforhealth.gov.au/sites/default/files/files/the guidelines/n55g_adult_brochure.pdf (accessed 15 Apr 2017).

39. Australian Government Department of Health. Australia's physical activity and sedentary behaviour guidelines. http://www.health. gov.au/internet/main/publishing.nsf/content/F01F92328EDADA 5BCA257BF0001E720D/\$File/brochure\%20PA\%20Guidelines A5 18-64yrs.PDF (accessed 15 Apr 2017).

40. Royal Australian College of General Practitioners (RACGP). Genera practice management of type 2 diabetes 2016-2018. http://www. racgp.org.au/your-practice/guidelines/diabetes/ (accessed $15 \mathrm{Apr}$ 2017).

41. Schulz K, Altman D, Moher D. CONSORT 2010 statement: updated guidelines for reporting parallel group randomised trials. J Clin Epidemiol 2010;340:c332.

42. Armstrong RA. When to use the Bonferroni correction. Ophthalmic Physiol Opt 2014;34:502-8.

43. Glickman ME, Rao SR, Schultz MR. False discovery rate control is a recommended alternative to Bonferroni-type adjustments in health studies. J Clin Epidemiol 2014;67:850-7.

44. Hauschke D, Steinijans VW. Directional decision for a two-tailed alternative. J Biopharm Stat 1996;6:211-3.

45. Zhang J, Boos DD. Mantel-haenszel test statistics for correlated binary data. Biometrics 1997;53:1185-98.

46. Hardin J, Hilbe J, Equations GE. Generalized Estimating Equations. Boca Raton, Florida, USA: Chapman and Hall, CRC Press, 2003.

47. Little RJA. A test of missing completely at random for multivariate data with missing values. J Am Stat Assoc 1988;83:1198-202.

48. Dziura JD, Post LA, Zhao Q, et al. Strategies for dealing with missing data in clinical trials: from design to analysis. Yale $\mathrm{J}$ Biol Med 2013;86:343.

49. Harris PA, Taylor R, Thielke R, et al. Research electronic data capture (REDCap)-a metadata-driven methodology and workflow process for providing translational research informatics support. J Biomed Inform 2009;42:377-81.

50. Barber JA, Thompson SG. Analysis of cost data in randomized trials: an application of the non-parametric bootstrap. Stat Med 2000;19:3219-36. 